\title{
Throughput-efficient Joint Coalition Formation and Bandwidth Allocation in Cognitive Radio Networks
}

\author{
Raza Umar ${ }^{1}$ and Wessam Mesbah ${ }^{1,2}$ \\ ${ }^{1}$ Electrical Engineering Department, King Fahd University of Petroleum \& Minerals, Dhahran, Saudi Arabia \\ ${ }^{2}$ Alexandria University, Alexandria, Egypt \\ Emails: $\{$ razaumar, mesbahw\} @kfupm.edu.sa
}

\begin{abstract}
In this paper we consider the problem of joint coalition formation and bandwidth allocation in cognitive radio networks. We assume that the secondary links will be partitioned into disjoint coalitions, by which the available spectrum will be reused. On the other hand, we assume that the members of each coalition will transmit over orthogonal sub-bands with the available spectrum being optimally allocated among them. We formulate the problem as a coordinated coalition formation game where the optimization is held at a secondary coordinator (SC) that has all the required channel information. Given these assumptions, we use a closed form expression of the optimal bandwidth allocation for any given network partition, and we propose an algorithm to reach a Nash-stable partition (coalition structure), with the objective of maximizing the network throughput. Performance analysis shows that the proposed coalition formation algorithm with optimal bandwidth allocation provides a substantial gain in the network throughput over existing coalition formation techniques as well as the simple cases of singleton and grand coalition.
\end{abstract}

Index Terms-Cognitive radio, Rate maximization, Coalition Formation, Nash-stable partition, Optimal bandwidth allocation.

\section{INTRODUCTION}

Cognitive radio networks (CRNs) based on dynamic spectrum access has emerged as a promising technology to meet the insatiable demand for radio spectrum by the emerging wireless applications [1]. The practical implementation of CRNs is facing many challenges and conflicting requirements such as primary user (PU) protection and secondary user (SU) rate maximization [2]. While the last decade of research activities has focused on enhancing spectrum sensing (SS) performance [3], or more recently on jointly optimizing the spectrum sensing and spectrum access parameters [4], [5] as a means of improving the secondary network throughput, their scope has proven to be limited after the FCC ruling [6] which obviated the SS requirement in CRNs. As a result, there has been a dire need to explore stand-alone efficient spectrum access schemes in a competitive environment where SUs do not solely rely on SS performance for their throughput improvement.

Hence, in this paper, we propose a game-theoretic strategy to dynamically share the available spectrum resources among competing SUs under the assumption that available spectrum opportunities are known a priori. Specifically, we propose to formulate the throughput-efficient network partitioning problem as a coordinated coalition formation (CF) game with exter- nalities and solve it with optimal bandwidth (BW) allocation.

\section{A. Related work and Our contributions}

Coalitional game-theoretic tools [7] have been explored for performance improvement of cognitive networks from different viewpoints [8]-[11]. However, the contribution of this paper differs from the existing work in number of ways. First, most of these works do not consider the overall network performance (such as total achievable sum-rate) as the objective function. Furthermore, the role of externalities (effect of SUs outside the coalition) in CF process is often ignored. For instance, a generic rate allocation problem has been analyzed in [8] as a CF game in characteristic form (without considering the effect of externalities) with transferable utility (TU) which allows the coalition value (coalition sum-rate) to be arbitrarily apportioned among the coalition members. In contrast, in this paper, we aim at maximizing the secondary network throughput by forming disjoint coalitions such that the total available transmission BW is made available to each coalition, while this BW is optimally sub-divided into orthogonal bands within each coalition. This requires us to model the $\mathrm{CF}$ game in a partition form since the payoff (transmission rate) of each coalition member is affected by the interference from simultaneous transmissions of members of different coalitions over the same frequency band. Moreover, the proposed game has non-transferable utility (NTU), since the optimal BW allocation restricts the distribution of the coalition value among the coalition members.

The coalitional games in partition form were recently investigated in [9], [10]. The authors in [9] considered the model in which coalition members shared the available BW according to their channel gain ratios, while in [10], sensing and access problems were jointly modeled where the competing SUs shared the spectrum in chunks of pre-fixed/slotted BW (as defined by PU channels). Also in [11], the available spectrum for the secondary communications is equally divided among the SUs. It is evident that these are not optimal BW allocation approaches from network rate perspective.

Furthermore, since distributed CF approaches suffer from prohibitively large information exchange [12] among the players proposing to form a coalition and sharing local channel state information (CSI), we address this problem here by considering a coordinated $\mathrm{CF}$ approach. The coordinated CF scheme devises a master controller node in the secondary 
network known as secondary coordinator (SC). Since our objective is to optimize the overall network performance, the proposed $\mathrm{CF}$ algorithm is designed to be executed centrally at the SC, which would otherwise yield CF to be practically un-realizable specifically for time limited, power limited, and control channel BW limited networks.

Therefore, our contribution on throughput-efficient spectrum sharing is fundamentally different from the existing work from two perspectives: (1) In comparison to conventional approach where the available spectrum resources are shared in terms of channels of pre-fixed and equal BW, we propose a continuous BW allocation among the coalition members and provide a closed form expression of rate optimal BW allocation. (2) We propose an efficient coordinated $\mathrm{CF}$ algorithm for the secondary network throughput maximization by considering a CF game with externalities and NTU, and analytically prove that the proposed algorithm would converge to a throughputefficient Nash-stable network partition.

The organization of this paper is as follows. Section II introduces the network model and identifies the two avenues we explored to enhance the rate of cognitive radio networks. The optimal BW allocation for a given network partition is presented in Section III while the algorithmic details of CF process to reach a rate-maximizing Nash-stable partition are discussed in Section IV. Performance analysis of the proposed algorithm is provided in Section V and finally the paper is concluded in Section VI.

\section{NETWORK MODEL}

We consider a cognitive radio network with $N$ secondary links (secondary transmitter-receiver (ST-SR) pairs), and a centrally located secondary coordinator (SC) node that is assumed to have CSI between all the secondary transmitters and secondary receivers. The role of the SC node is only to manage the coalition formation and the BW allocation between the different secondary links. We assume that the total available BW for secondary access is $W \mathrm{~Hz}$, and we assume that the channel between any of the secondary transmitters and any of the secondary receivers over this BW follows a quasistatic flat fading model. A representative network model with a centrally located SC and randomly distributed ST-SR pairs in its coverage area, forming a representative network partition, is illustrated in Fig. 1.

The throughput-efficient network partitioning problem is addressed from two perspectives. First, we propose frequency reuse of the available BW by partitioning the $N$ links into a set of coalitions such that each coalition will be using the total available BW. Such partitioning would, on one hand, have the potential to increase the network rate because of reusing the $\mathrm{BW}$, but would on the other hand cause interference between the different coalitions. Therefore, the choice of the partitioning structure would substantially affect the total achievable network rate, and hence in this paper we propose a CF algorithm that can be used to reach the Nash-stable network partition. Second, since each coalition in the network partition will be using the total available $\mathrm{BW}(\mathrm{W} \mathrm{Hz})$, we propose

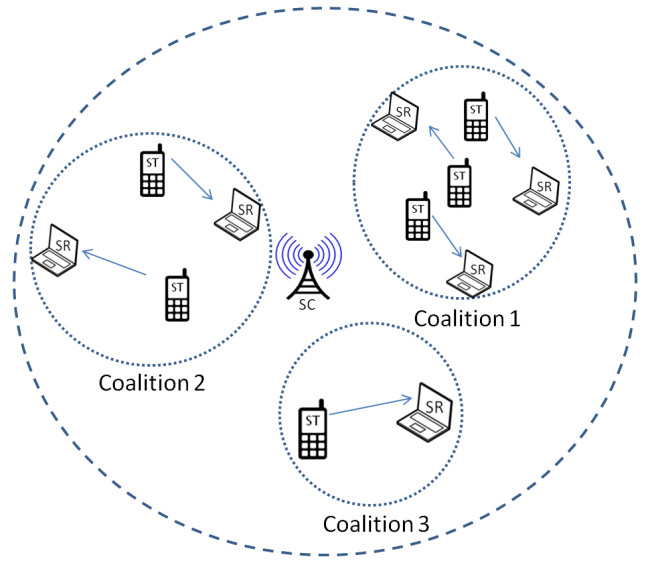

Fig. 1. A representative network model with SC and ST-SR pairs.

to optimally allocate this BW among the coalition members based on the available CSI with the objective of maximizing the total network throughput. It is important to highlight here that the two problems discussed above are coupled, which means that none of them can be solved without solving the other. This is because we can not form the coalitions without knowing the optimal rate of each coalition, and at the same time the optimal rate of each coalition depends on which players are inside this coalition and which ones are outside it. This makes the problem hard but interesting. We start our analysis by solving the optimal $\mathrm{BW}$ allocation problem for a given network partition, and then we discuss how to exploit this optimal bandwidth allocation in the proposed coalition formation algorithm.

\section{Optimal BANDWIDTH ALLOCATION}

In this section we discuss the optimal bandwidth allocation among the members of a coalition for a given network partition. Let the network partition at a certain time instant consists of $|\Pi|$ disjoint coalitions, i.e. $\Pi=\left\{S_{1}, S_{2}, \ldots, S_{|\Pi|}\right\}$. In the system model under consideration, each of the $|\Pi|$ coalitions will use the total available bandwidth $W$. Consider Coalition $S_{k} \in \Pi$, with $\left|S_{k}\right|$ representing the number of members (links) in $S_{k}$. We will use $m_{i}^{k}$ to refer to Member (Link) $i$ of Coalition $S_{k}$ while $P_{i}^{k}$ will represent the transmission power of this member. Each member $m_{i}^{k}$ of $S_{k}$ will be allocated a fraction $\mu_{i}^{k}$ of the total bandwidth, where $\sum_{i=1}^{\left|S_{k}\right|} \mu_{i}^{k}=1$. The total interference power affecting the bandwidth $W$ being used by Coalition $S_{k}$ is the sum of all received power from all members in all other coalitions. For simplicity of the analysis, we approximate the interference affecting the band $\mu_{i}^{k} W$ being used by Member $m_{i}^{k}$ to be the average interference affecting this band which is a fraction $\mu_{i}^{k}$ of the total interference power affecting the total bandwidth at the receiver of Link $m_{i}^{k}$, i.e.,

$$
\begin{aligned}
\bar{I}_{i}^{k} & =\mu_{i}^{k} \sum_{l \neq k} \sum_{j=1}^{\left|S_{l}\right|} P_{j}^{l}\left|h_{j i}^{l k}\right|^{2} \\
& =\mu_{i}^{k} I_{i}^{k}
\end{aligned}
$$


where $h_{j i}^{l k}$ is the channel between the transmitter of Link $j$ in Coalition $S_{l}$ and the receiver of Link $i$ in Coalition $S_{k}$, and hence the total rate of Coalition $S_{k}$ can be written as

$$
\mathcal{R}^{S_{k}}=\sum_{i=1}^{\left|S_{k}\right|} R_{i}^{S_{k}}=\sum_{i=1}^{\left|S_{k}\right|} \mu_{i}^{k} W \log \left(1+\frac{P_{i}^{k}\left|h_{i i}^{k k}\right|^{2}}{\mu_{i}^{k}\left(N_{0} W+I_{i}^{k}\right)}\right) .
$$

In order to maximize the rate achieved by this coalition, the problem can be formulated as

$$
\begin{aligned}
& \max _{\mu_{i}^{k}} \sum_{i=1}^{\left|S_{k}\right|} \mu_{i}^{k} W \log \left(1+\frac{P_{i}^{k}\left|h_{i i}^{k k}\right|^{2}}{\mu_{i}^{k}\left(N_{0} W+I_{i}^{k}\right)}\right), \\
& \text { subject to } \sum_{i=1}^{\left|S_{k}\right|} \mu_{i}^{k}=1 \text { and } 0 \leq \mu_{i}^{k} \leq 1 .
\end{aligned}
$$

It can be shown that this problem is concave, and hence the globally optimal solution is guaranteed to be obtained numerically. However, here we provide a closed form for the optimal solution as following:

The objective function of (3) can be written as

$$
W \sum_{i=1}^{\left|S_{k}\right|} \mu_{i}^{k} \log \left(1+\frac{x_{i}^{k}}{\mu_{i}^{k}}\right)
$$

where $x_{i}^{k}=\frac{P_{i}^{k}\left|h_{i i}^{k k}\right|^{2}}{N_{0} W+I_{i}^{k}}$. Since the log function is concave, and since $\sum_{i=1}^{\left|S_{k}\right|} \mu_{i}^{k}=1$ and $0 \leq \mu_{i}^{k} \leq 1$, then the objective function can be upper bounded by

$$
\begin{aligned}
W \sum_{i=1}^{\left|S_{k}\right|} \mu_{i}^{k} \log \left(1+\frac{x_{i}^{k}}{\mu_{i}^{k}}\right) & \leqslant W \log \left(1+\sum_{i=1}^{\left|S_{k}\right|} \mu_{i}^{k} \frac{x_{i}^{k}}{\mu_{i}^{k}}\right), \\
& =W \log \left(1+\sum_{i=1}^{\left|S_{k}\right|} x_{i}^{k}\right) .
\end{aligned}
$$

It can be shown that this upper bound can be achieved by choosing

$$
\mu_{i, \mathrm{opt}}^{k}=\frac{x_{i}^{k}}{\sum_{m=1}^{\left|S_{k}\right|} x_{m}^{k}}
$$

Hence, the closed form expression of optimal rate $R_{i, o p t}^{S_{k}}$ for any $i \in S_{k}$ is given by:

$$
R_{i, \mathrm{opt}}^{S_{k}}=\mu_{i, \mathrm{opt}}^{k} W \log \left(1+\sum_{m=1}^{\left|S_{k}\right|} x_{m}^{k}\right) .
$$

\section{COORDINATED COALITION FORMATION}

In the previous section, we derived a closed form expressions of the rate optimal BW allocation among coalition members under the assumption that a network partition is known apriori. In this section, we address the problem of finding a Nash-stable partition by answering the key question: In order to maximize the network throughput, should a player act in a non-cooperative manner and utilize the total available $W$ $\mathrm{Hz}$ or make a coalition with other player(s) and divide $W \mathrm{~Hz}$ among the coalition members? It is important to highlight that a non-cooperative approach (singleton coalitions: coalitions consisting of one player only) offers a total bandwidth of $W \mathrm{~Hz}$ to each player and provides maximum spectrum reuse in the network at the cost of high interference among all the players. On the other hand, grand coalition avoids the interference by dividing the available BW among all players with no spectrum re-use. For the considered throughput maximization problem, a balance needs to be maintained between interference avoidance and spectrum re-use, and the optimal network partition might neither be singleton nor grand [12]. However, finding an optimal network partition was shown to be NP-complete [13], as the number of possible partitions (given by Bell number $B_{N}$ [14]) grows exponentially with the number of communication links, $N$, in the network. Hence, there is a need to develop algorithms to organize links into non-overlapping coalitions that are at least stable, if not optimal. Further, since we aim at improving upon the overall system performance in the form of network rate, the stable partition search is assumed to be executed centrally at SC node by playing a coordinated coalition formation game.

\section{A. Game formulation:}

We model the problem of finding a throughput-efficient Nash-stable network partition, as a CF game in partition form with non-transferable utility (NTU), defined as follows [7]:

Definition 1: A CF game in partition form with NTU is specified by a pair $(\mathcal{N}, \mathcal{V})$, where $\mathcal{N}$ is the set of players, and $\mathcal{V}$ is a mapping such that for every partition $\Pi \in \mathcal{P}$, where $\mathcal{P}$ is the set of all possible partitions of $\mathcal{N}$, and every coalition $S_{k} \subseteq \mathcal{N}, S_{k} \in \Pi, \mathcal{V}\left(S_{k}, \Pi\right)$ assigns a set of $\left|S_{k}\right|$-dimensional vectors, representing the possible payoffs that the players in $S_{k}$ can achieve when the partition $\Pi$ is in place. Formally, $\mathcal{V}\left(S_{k}, \Pi\right)$ is a closed, convex subset of $\mathbb{R}^{\left|S_{k}\right|}$.

In the proposed CF game, the $N$ secondary links act as the players of the game constituting the set $\mathcal{N}=\{1,2, \ldots, N\}$. For any coalition $S_{k} \subseteq \mathcal{N}, S_{k} \in \Pi, \mathcal{V}\left(S_{k}, \Pi\right)$ contains only a single vector $\mathbf{v}\left(S_{k}, \Pi\right) \in \mathbb{R}^{\left|S_{k}\right|}$ where each element $v_{i} \in$ $\mathbf{v}\left(S_{k}, \Pi\right)$ represents the payoff of player $i \in S_{k}$ and is given by its respective rate $R_{i, o p t}^{S_{k}}$ as:

$\mathcal{V}\left(S_{k}, \Pi\right)=\left\{\mathbf{v}\left(S_{k}, \Pi\right) \in \mathbb{R}^{\left|S_{k}\right|} \mid v_{i}\left(S_{k}, \Pi\right)=R_{i, o p t}^{S_{k}}\right\}$

where, $R_{i, \text { opt }}^{S_{k}}$ is given by (7). Since the value of any coalition $S_{k}$ cannot be arbitrarily divided among the coalition members, the proposed CF game has a non-transferable utility. Furthermore, the CF game is in partition form since the payoff of every player $i \in S_{k} ; R_{i, o p t}^{S_{k}}$, depends not only on the players in $S_{k}$ but also on the players in $\mathcal{N} \backslash S_{k}$ which interfere with $i \in S_{k}$.

In the next subsection, we describe the proposed algorithm to reach a Nash-stable network partition.

\section{B. CF algorithm:}

Let the current network partition be $\Pi=\left\{S_{1}, S_{2}, \ldots, S_{|\Pi|}\right\}$. The sum of total rates of all $(|\Pi|)$ coalitions under the partition $\Pi$; i.e. $\Re^{\Pi}=\sum_{k=1}^{|\Pi|} \mathcal{R}^{S_{k}}$, where $\mathcal{R}^{S_{k}}$ is the total rate of Coalition $S_{k}$ and is defined in (2), represents the network rate 
TABLE I

CF ALGORITHM DESIGNED FOR NETWORK RATE BASED SWITCH RULE

\begin{tabular}{l} 
Algorithm: $\mathrm{CF}$ algorithm is executed centrally at SC node where, in \\
each round, the algorithm goes sequentially over all players $i \in \mathcal{N}$. \\
\hline Initialization: \\
Initial partition: $\Pi_{\text {init }}=\{\{1\},\{2\}, \ldots,\{N\}\}$. Initial rate: $\Re^{\Pi_{\text {init }}}$. \\
The two phases of proposed CF algorithm: \\
Phase 1: Starting from current partition $\Pi$ (at the beginning of \\
all time, $\Pi=\Pi_{\text {init }}$ ) and based on action space $A_{i}$ of player $i$, the \\
algorithm iterates over all players $i \in \mathcal{N}$ to find a set of all partitions \\
$\mathcal{P}_{\Pi}$ reachable from $\Pi$ via one switch operation by any of the players. \\
Phase $2: \Pi^{*} \in \mathcal{P}_{\Pi}$ giving the maximum network rate is identified \\
and the transition $\Pi \rightarrow \Pi^{*}$ is made provided $\Re^{*}>\Re^{\Pi}$. \\
The algorithm converges to a throughput-efficient, stable, final \\
network partition $\Pi_{f}$, if, after iterating over all the players $i \in \mathcal{N}$, \\
no transition is made; i.e. every player prefers to stay in its current \\
coalition.
\end{tabular}

when partition $\Pi$ is in place. Hence, the achievable throughput by the cognitive radio network strongly depends on how the secondary links are organized into coalitions.

The proposed CF algorithm is summarized in Table I. We initialize the algorithm with a non-cooperative setup (exploiting full frequency re-use) where the initial network partition $\Pi_{\text {init }}$ is composed of $N$ singleton coalitions as: $\Pi_{\text {init }}=\{\{1\},\{2\}, \ldots,\{N\}\}$ and $\Re^{\Pi_{\text {init }}}$ is calculated as the initial network rate. The algorithm consists of two phases and goes sequentially, in a round robin fashion, over all players $i \in \mathcal{N}$ to examine which action, a player can take to improve the total network rate. The action space of player $i$ is defined as: $A_{i}=\{$ stay, switch $\} \forall i \in \mathcal{N}$. Hence, a player $i$ can stay in its current coalition with associated partition $\Pi$ or it may decide to switch to another coalition (which might even be an empty coalition $\phi$, which means that a player may decide to leave and act as a singleton) leading to a new network partition $\Pi$. In this way, the proposed switch operation updates the partition structure by either keeping the same number of coalitions in the network partition or increasing/decreasing the number of coalitions by only 1 , and hence, provides a mechanism to make a transition from one network partition to another. It is important to point out here that, unlike existing CF algorithms, [5], [10], wherein a player $i \in S_{k}$ arbitrarily selects a coalition $S_{l}$ to investigate the possibility of performing a switch operation and it actually leaves its current coalition $S_{k}$ to join $S_{l}$ if a pre-defined switch rule is satisfied, we propose a two-phase $\mathrm{CF}$ algorithm that examines all switch/stay possibilities of all the players, at each $\mathrm{CF}$ round, before performing the action that would maximize the network throughput in this round. In phase one, we identify, at each $\mathrm{CF}$ round, all the feasible partitions $\mathcal{P}_{\Pi}$, which can be reached from $\Pi$ via only one switch operation by any one player in the network. In phase two, the network partition $\Pi^{*} \in \mathcal{P}_{\Pi}$ giving the maximum network rate is identified and the transition from partition $\Pi$ to the new partition $\Pi^{*}$ is executed only if the network throughput achieved under partition $\Pi^{*}$; i.e., $\Re^{\Pi^{*}}$, is more than the current network rate $\Re^{\Pi}$, otherwise, the current network partition $\Pi$ is maintained. Formally the transition rule is defined as:

Definition 2: Given the network partition $\Pi=$ $\left\{S_{1}, S_{2}, \ldots, S_{|\Pi|}\right\}$ of $\mathcal{N}$, a player $i \in \mathcal{N}$ decides to switch from its current coalition $S_{k} \in \Pi$ to join another coalition $S_{l} \in \Pi \cup \phi$ for $l \neq k$, hence forming $\Pi^{*}=\left\{\Pi \backslash\left\{S_{k}, S_{l}\right\}\right\} \cup$ $\left\{S_{k} \backslash\{i\}\right\} \cup\left\{S_{l} \cup\{i\}\right\}$ where $\Pi^{*} \in \mathcal{P}_{\Pi}$, according to the following switch/transition rule:

$$
\Pi \rightarrow \Pi^{*} \Longleftrightarrow \Re^{\Pi^{*}}>\Re^{\Pi} \forall \Pi \in \mathcal{P}_{\Pi} \text { and } \Pi \neq \Pi^{*}
$$

Each round of the proposed $\mathrm{CF}$ algorithm concludes with a single (best possible) switch/stay operation.

Remark 1: The algorithm reaches a stable throughputefficient network partition $\Pi_{f}$, if, after iterating over all the players $i \in \mathcal{N}$, no transition is made; i.e. each player prefers to stay in its current coalition.

Remark 2: The proposed CF algorithm is repeated periodically throughout the network operation to handle environmental changes such as mobility or joining/leaving of players.

\section{Convergence, throughput efficiency and stability of final network partition:}

For any current network partition $\Pi$, the proposed switch rule ensures that in each round of the $\mathrm{CF}$ algorithm, the switch operation compares all possible partitions in $\mathcal{P}_{\Pi}$ and, if executed, always results in a new network partition $\Pi^{*} \in \mathcal{P}_{\Pi}$ with increased network throughput (see Equation 9). This guarantees oscillation avoidance, which means that the $\mathrm{CF}$ algorithm cannot go back to an already visited network partition. Furthermore, for finite number of secondary links $N$, the number of possible partitions is also countably finite (given by Bell number $B_{N}$ [14]), which guarantees that the $\mathrm{CF}$ algorithm always converges to a stable throughput-efficient network partition $\Pi_{f}$ after finite number of rounds/iterations. The throughput efficiency of this resulting network partition $\Pi_{f}$ is evident from the fact that the network throughput is increased in each round of the CF algorithm after every switch operation. As a result, the proposed algorithm converges to a final network partition $\Pi_{f}$ which offers the throughput that cannot be further increased by making transition to any partition that belongs to $\mathcal{P}_{\Pi_{f}}$, and hence partition $\Pi_{f}$ is a Nash-stable partition defined as follows:

Definition 3: A partition $\Pi=\left\{S_{1}, S_{2}, \ldots, S_{|\Pi|}\right\} \forall i \in \mathcal{N}$ with a rate $\Re^{\Pi}$ is said to be Nash-stable if there does not exist any partition $\Pi^{*} \in \mathcal{P}_{\Pi}$ such that $\Re^{\Pi^{*}}>\Re^{\Pi}$.

\section{Performance Analysis}

In this section, we analyze the performance of the proposed $\mathrm{CF}$ algorithm by comparing the average network rate (bits/sec) with two benchmark network partitions: (1) Non-cooperative solution comprising of all singleton coalitions and (2) Fully cooperative solution resulting in a single grand coalition. We assume that the total available bandwidth $W$ for the secondary access is $5 \mathrm{MHz}$. All the channels are assumed to follow a quasi-static Rayleigh flat fading model, and hence the received signal power, interference power and signal to noise ratio (SNR) are exponentially distributed, and the results 
are averaged over 100,000 channel realizations. The transmit power $P_{i}$ and noise power spectral density $\left(N_{0}\right)$ are normalized to 1 , and their effects are included in the channel coefficients. We assume the mean of the interference power among the neighboring links to be uniformly distributed between $0 d B$ and $10 d B$ while comparatively much lower interference, with means uniformly distributed between $-10 d B$ and $-5 d B$, is assumed to be originating from far away links.

\section{A. Average network throughput comparison with slotted BW allocation and distributed CF approaches}

Fig. 2 highlights the average network throughput improvement offered by the proposed $\mathrm{CF}$ algorithm with optimal BW allocation over the cases of always singleton/grand in addition to two schemes popularly used in literature: (1) when the spectrum is shared among competing players in the form of channels of pre-fixed BW (slotted channels) [4], and (2) when $\mathrm{CF}$ is based on the selfish preferences of players [5] and [10]. It is worth noting that [4], [5] and [10] all consider the joint optimization of sensing time and secondary transmission rate, and hence their results cannot be compared directly to ours. However, Fig. 2 shows the average network throughput improvement offered by the proposed CF algorithm over slotted BW allocation and over selfish CF approaches applied to our network model. The two selfish CF algorithms compared are: (1) self-no-no as proposed in [5] where the switch rule can be viewed as a selfish decision made by a player to move from its current coalition to a new coalition, regardless of the effect of its move on other players and (2) self-indv-no as given in [10] where a player decides to switch to a new coalition if it can strictly improve its own rate, without decreasing the rate of any member of the new coalition.

Our results indicate that at very low/high average direct link SNR values, the proposed CF algorithm converges to grand/singleton structure, respectively, while for moderate SNR values $(0 d B<S N R<15 d B)$, it provides a substantial network rate gain over always singleton/grand structure. For example, at $S N R=5 d B$, the proposed CF algorithm offers $42.5 \%$ more network rate as compared to always singleton/grand structure. Furthermore, the proposed CF algorithm provides $15 \%$ throughput improvement when compared with selfish CF algorithms (self-no-no and selfindv-no) with optimal $\mathrm{BW}$ allocation and $10.5 \%$ throughput improvement over using slotted BW allocation in the proposed $\mathrm{CF}$ algorithm. Additionally, as the average direct link SNR increases above $10 \mathrm{~dB}$, selfish CF based on self-no-no switch rule starts to diverge from the expected final network partition with all singleton Coalitions resulting in much lower network throughput.

\section{B. Effect of number of links on average network throughput}

Fig. 3 shows the average network throughput improvement by employing the proposed joint $\mathrm{CF}$ and optimal $\mathrm{BW}$ allocation algorithm for various network sizes operating at average direct link $S N R=5 d B$. These results indicate that the gain

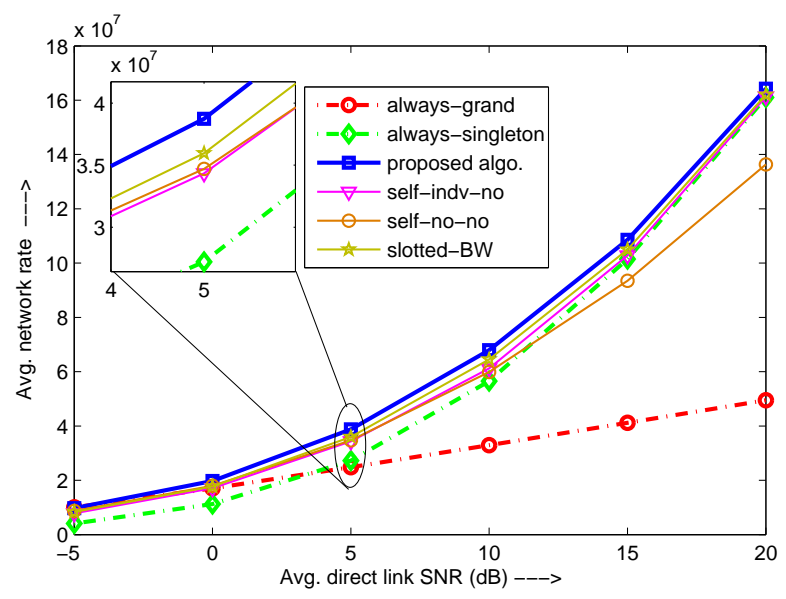

Fig. 2. Average network rate comparison with conventional distributed $\mathrm{CF}$ with optimal $\mathrm{BW}$ and centralized $\mathrm{CF}$ with slotted $\mathrm{BW}$ allocation for 10 links.

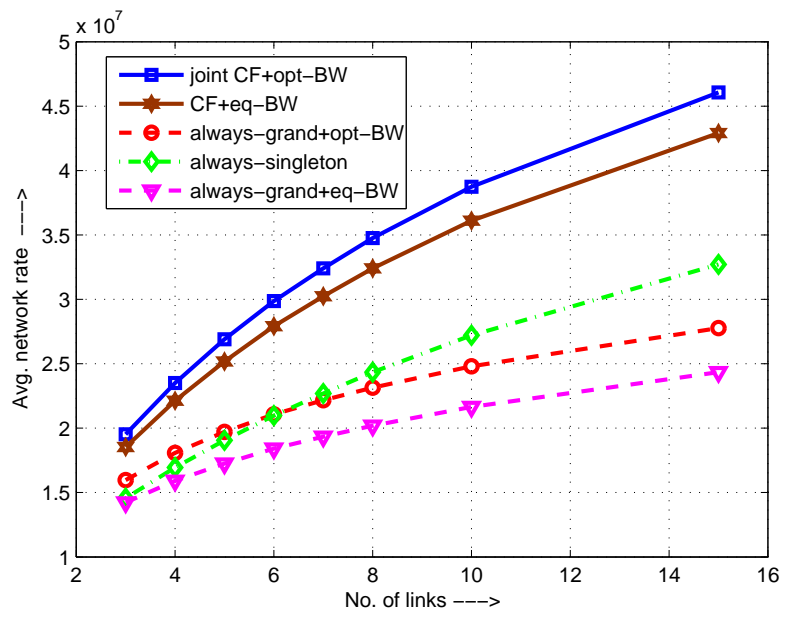

Fig. 3. Average network rate improvement by using joint $\mathrm{CF}+\mathrm{BW}$ allocation algorithm for different network sizes with average direct link SNR $=5 d B$.

in the network average throughput, offered by the proposed algorithm, increases as the network size increases. Furthermore, Fig. 3 quantifies separately the gains achieved by the proposed $\mathrm{CF}$ algorithm and by the optimal BW allocation. Our results show that for $N=10$ links, the proposed $\mathrm{CF}$ algorithm with equal BW allocation offers $32.8 \%$ more average network throughput, as compared to the always singleton case, which is further increased by $9.7 \%$, reaching up to $42.5 \%$ when the optimal bandwidth allocation is employed.

\section{Convergence speed and computational complexity}

The proposed CF algorithm exploits the computational capabilities of the SC to converge to a final Nash-stable network partition. The actual convergence speed strongly depends on the initial network partition $\Pi_{\text {init }}$ and average direct link SNR. The left axis of Fig. 4 depicts the convergence speed in terms of $\mathrm{CF}$ rounds for $N=10$ links when the proposed $\mathrm{CF}$ algorithm is initialized in a singleton/grand state and operated over a wide range of average direct link SNR. For very low direct link SNRs, most of the links experience high interference and hence starting from singleton/grand, prefer to 


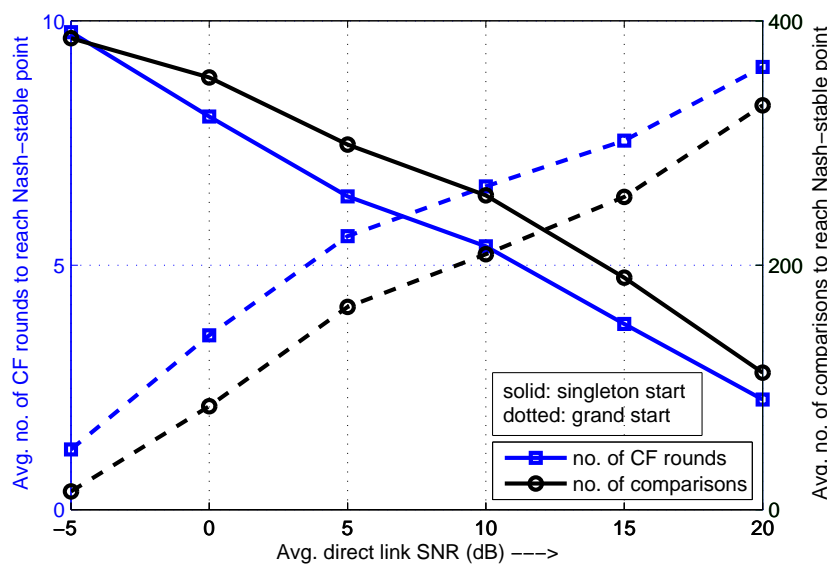

Fig. 4. Average convergence speed (number of $\mathrm{CF}$ rounds) and average number of network rate comparisons made to reach a Nash-stable partition.

reach a grand coalition. Since each $\mathrm{CF}$ round incorporates one switch action, starting from singleton, $N$ links require $N-1$ switch operations to reach a grand coalition, while starting from a grand coalition, all the $N$ links prefer to stay in a grand coalition. It can be seen from Fig. 4 that at $S N R=-5 d B$, starting from singleton requires an average number of rounds that is slightly greater than $N-1=9$ to converge, while starting from a grand coalition requires an average number of rounds that is slightly more than 1 to converge. This reflects that at an average direct link $S N R=-5 \mathrm{~dB}$, for certain realistic channel realizations, grand coalition does not emerge as the throughput-efficient network partition. On the other hand, as the average direct link SNR is increased (relative to the interference), it is expected that the links would tend to operate as singleton. Therefore, the number of $\mathrm{CF}$ rounds required to reach a Nash-stable network partition decrease/increase with improving average direct link SNR, for the cases of starting from singleton/grand, respectively.

The algorithm complexity in terms of total network rate comparisons, made before converging to a Nash-stable network partition, is depicted through the right axis of Fig. 4. In a given $\mathrm{CF}$ round, the maximum number of rate comparisons is $N(N-1) / 2$ which occurs when the current network partitions comprises of all singleton coalitions, which gives a worst case complexity of $\mathcal{O}\left(N^{2}\right)$. However, as coalitions start to form, this complexity becomes smaller. On the other hand, the minimum rate comparisons are $N$ which occurs when the current partition is a grand coalition leading to the best case complexity of $\mathcal{O}(N)$. Therefore, the complexity in terms of rate comparisons made during a $\mathrm{CF}$ round lie between the two extremes. This is evident from Fig. 4 which shows that algorithm complexity, in terms of number of network rate comparisons, is proportional to the number of $\mathrm{CF}$ rounds, where the average number of rate comparisons per $\mathrm{CF}$ round varies between $[10,40]$.

\section{CONCLUSIONS}

In this paper, we applied coalitional game theoretic framework to spectrum sharing problem in cognitive radio networks and studied the effect of network size and average direct link SNR on achievable network rate. We formulated the throughput-efficient network partitioning problem as a coordinated CF game and proposed an algorithm to organize communication links into Nash-stable, throughput-efficient network partition while maintaining the crucial balance between interference avoidance and spectrum re-use. We showed that the throughput-efficient network partition converges to a grand coalition in an interference rich environment when the average direct link SNR is very low, while for high average direct link SNR values, the network contains only singleton coalitions. Further, we showed that at moderate SNR values, significant network rate improvement over grand/singleton coalitions is achieved through the proposed CF algorithm which can be further enhanced through an optimal BW allocation for which the closed form expression has also been derived.

\section{REFERENCES}

[1] I. F. Akyildiz, W. Y. Lee, M. C. Vuran, and S. Mohanty, "NeXt generation/dynamic spectrum access/ cognitive radio wireless networks: A survey," Computer Networks J., vol. 50, no. 13, pp. 2127-2159, 2006.

[2] R. Umar, and A. U. H. Sheikh, "Cognitive radio oriented wireless networks: challenges and solutions," IEEE Int. Conf. on Multimedia Comp. and Sys. (ICMCS'12), pp. 992-997, Morocco, May 10-12, 2012.

[3] R. Umar, and A. U. H. Sheikh, "A comparative study of spectrum awareness techniques for cognitive radio oriented wireless networks," Elsevier Physical Communications J. Special Issue: Cognitive Radios, vol. 9, pp. 148-170, Dec. 2013.

[4] Z. Khan, J. Lehtomaeki, M. Codreanu, M. Latva-aho, and L. A. DaSilve, "Throughput efficient dynamic coalition formation in distributed cognitive radio networks," EURASIP J. on Wireless Communications and Networking, vol. 2010, article no. 87, April 2010.

[5] X. Hao, M. H. Cheung, V. W. S. Wong, and V. C. M. Leung, "Hedonic coalition formation game for cooperative spectrum sensing and channel access in cognitive radio networks," IEEE Trans. on Wireless Communications, vol. 11, no. 11, pp. 3968-3979, Nov. 2012.

[6] FCC, ET Docket No. 10-174 2nd memo. opinion and order, Sept. 2010.

[7] R. B. Myerson, Game Theory, Analysis of Conflict. Cambridge, MA: Harvard Univ. Press, Sept. 1991.

[8] R. La and V. Anantharam, "A game-theoretic look at the Gaussian multiaccess channel," DIMACS Series in Discrete Math. Theoretical Comp. Sci., vol. 66, pp.87106.

[9] Z. Khan, S. Glisic, L. DaSilva and J. Lehtomaki, "Modeling the dynamics of coalition formation games for cooperative spectrum sharing in an interference channel," Trans. on Computational Intelligence and AI in Games, vol. 3, no. 1, pp. 1730, 2011.

[10] W. Saad, Z. Han, R. Zheng, A. Hjorungnes, T. Basar, and H. V. Poor, "Coalitional games in partition form for joint spectrum sensing and access in cognitive radio networks", IEEE J. of Sel. Topics in Signal Processing, vol. 6, no. 2, pp. 195-209, 2012.

[11] Y. Yuan, P. Bahl, R. Chandra, P. A. Chou, J. I. Ferrell, T. Moscibroda, S. Narlanka, and W. Yunnan, "KNOWS: Cognitive Radio Networks Over White Spaces," IEEE DySPAN'07, pp. 416-427, April 2007.

[12] D. Ray, A Game-Theoretic Perspective on Coalition Formation. New York: Oxford Univ. Press, Jan. 2007.

[13] T. Sandholm, K. S. Larson, M. R. Andersson, O. Shehory, and F. Tohm, "Coalition structure generation with worst case guarantees," Artificial Intelligence, vol. 111, no. 1/2, pp. 209238, Jul. 1999.

[14] N. J. A. Sloane (Ed.), "Bell or exponential numbers: ways of placing $n$ labeled balls into $n$ indistinguishable boxes", The On-Line Encyclopedia of Integer Sequence (OEIS). 